\title{
Giant GIST of the Stomach Masquerading Gigantic Cyst Located in the Upper Part of the Peritoneal Cavity
}

\section{Ramiz B Bayramov*, Ramila T Abdullayeva and Simara E Huseynova}

Department of Oncology, Azerbaijan Medical University, Baku, Azerbaijan

*Corresponding Author: Ramiz B Bayramov, Department of Oncology, Azerbaijan Medical University, Baku, Azerbaijan.
Received: April 22, 2021

Published: May 11, 2021

(C) All rights are reserved by Ramiz B

Bayramov., et al.

\begin{abstract}
Gastrointestinal stromal tumors (GISTs) are uncommon when compared to all gastrointestinal neoplasms but are the most common (80 - 85\%) and specific mesenchymal tumors of the gastrointestinal tract. GISTs often present solid tumors and are rarely predominantly cystic. This report presents a case of a large exophytic cystic GIST in 61-year-old woman who was referred to specialized oncologic clinic from an unspecialized hospital with a suspicion of huge pancreatic cystic lesion based on CT imaging. A multidisciplinary meeting including gastrointestinal surgical oncologists, endoscopists and radiologists was held before operation and gastric GIST with cystic appearance was highly doubtful as a definite preoperative clinicoradiologic diagnosis. Radiologically other cystic lesions were less doubtful. Taking into consideration the possible clinicoradiological diagnosis surgery was planned. At laparotomy a large exophytic cystic mass was found to be attached by a thin $(3.0 \mathrm{~cm}$ in diameter $)$ stalk to the greater curvature of the stomach at the level of its upper third. No infiltration into the surrounding structures was observed. The cyst was very mobile and did not attach to adjacent anatomical structures by adhesions. The lowermost pole of the lesion was at the level lower than umbilicus. The size of the mass was $18 \times 17 \times 10 \mathrm{~cm}$. Wedge resection of the greater curvature of the stomach along with the cystic mass and the adherent greater omentum was performed. Pathology confirmed spindle cell tumor. Mitotic index was $\leq 5 / 50$ HPFs and the tumor cells demonstrated positivity for CD117 on immunohistochemical staining. The histological features together with the immunohistochemical profile of the tumor cells were consistent with the GISTs. The tumor was accepted as GIST of the stomach with high risk of recurrence according to the generally accepted classifications. Patient was administrated imatinib for 12 months and during the 16 months' postoperative period no local recurrence or metastases were detected.
\end{abstract}

Keywords: GIST; Gastric Cancer; Intraabdominal Cyst

\section{Background}

GISTs are uncommon when compared to all gastrointestinal neoplasms but are the most common (80 - 85\%) and specific mesenchymal tumors of the gastrointestinal tract $[1,4,6,11]$. GIST is a rare neoplasm that represents approximately $1.0 \%$ of all malignant neoplasms of the gastrointestinal tract with an annual incidence of approximately 4 per million people $[5,11,13]$.

Most GISTs arise from the stomach (50 - 62\%), the small intestine $(20-30 \%)$, the colon $(11 \%)$ and the rectum (7\%), while the esophagus is rarely involved (0.6 - 1\%) [5]. GISTs usually present as solid tumors of the gastrointestinal tract, mostly found in the stomach and intestine as mentioned above $[7,8,15,17]$. They rarely can also present as cystic lesions [7,8]. Proper identification of GISTs has become very important since the availability of a specific pathogenesis-targeted treatment, namely, imatinib [15].

We report a gigantic unilocular cystic gastric GIST with exophytic pedunculated growth that preoperatively diagnosed correctly in a specialized center.

\section{Case Presentation}

61-year-old woman was referred to Oncologic Clinic, Azerbaijan Medical University from an unspecialized hospital with a suspicion 
of huge pancreatic cystic lesion based on CT imaging. The patient complained of abdominal fullness and had no pain. She never had any previous attack of pancreatitis. Physical examination revealed a mobile palpable mass in the left upper quadrant of the abdomen. Upper gastrointestinal endoscopy detected no pathology. On reviewing CT images, a huge well-defined unilocular cystic mass located in left supracolic compartment was seen (Figure). There was no evidence of a connection between the cyst and the pancreas that looked normal with no radiological evidence of pancreatic abnormality denoting previous pancreatitis. The uppermost part of the cystic lesion had a small solid component that seemed to be extension of the gastric wall. The lesion had not intimate relations with other adjacent organs. CT findings demonstrated no vascularity of the lesion. Results of blood samples were within normal values. A multidisciplinary meeting including gastrointestinal surgical oncologists, endoscopists and radiologists was held before surgery and gastric GIST with cystic appearance was highly doubtful as a definite preoperative clinicoradiologic diagnosis. Radiologically other cystic lesions were less doubtful.

Considering the possible clinicoradiologic diagnosis surgery was planned. At laparotomy a large $(18 \times 17 \times 10 \mathrm{~cm})$ exophytic cystic mass was found to be attached by a thin $(3.0 \mathrm{~cm}$ in diameter) stalk to the greater curvature of the stomach at the level of its upper third. No infiltration into the surrounding structures was observed. The cyst was very mobile and did not attach to adjacent anatomical structures by adhesions. The lowermost pole of the lesion was at the level lower than umbilicus.

Wedge resection of the greater curvature of the stomach along with the cystic mass and the adherent greater omentum was performed. Grossly, it was an unilocular cystic tumor filled with thin fluid, with a smooth outer surface and a thick wall. The stalk was connecting the cystic tumor with the stomach and looked like an extension of the stomach wall.

Pathological examination of the cystic mass confirmed spindle cell tumor. Mitotic index was $\leq 5 / 50 \mathrm{HPFs}$ and the tumor cells demonstrated positivity for CD117 on immunohistochemical staining. The histological features together with the immunohistochemical profile of the tumor cells were consistent with GISTs.

The tumor was accepted as GIST of the stomach with high risk of recurrence according to the generally accepted classifications. Patient was administrated imatinib for 12 months and during the 16 months' postoperative period no recurrence or metastases were detected.
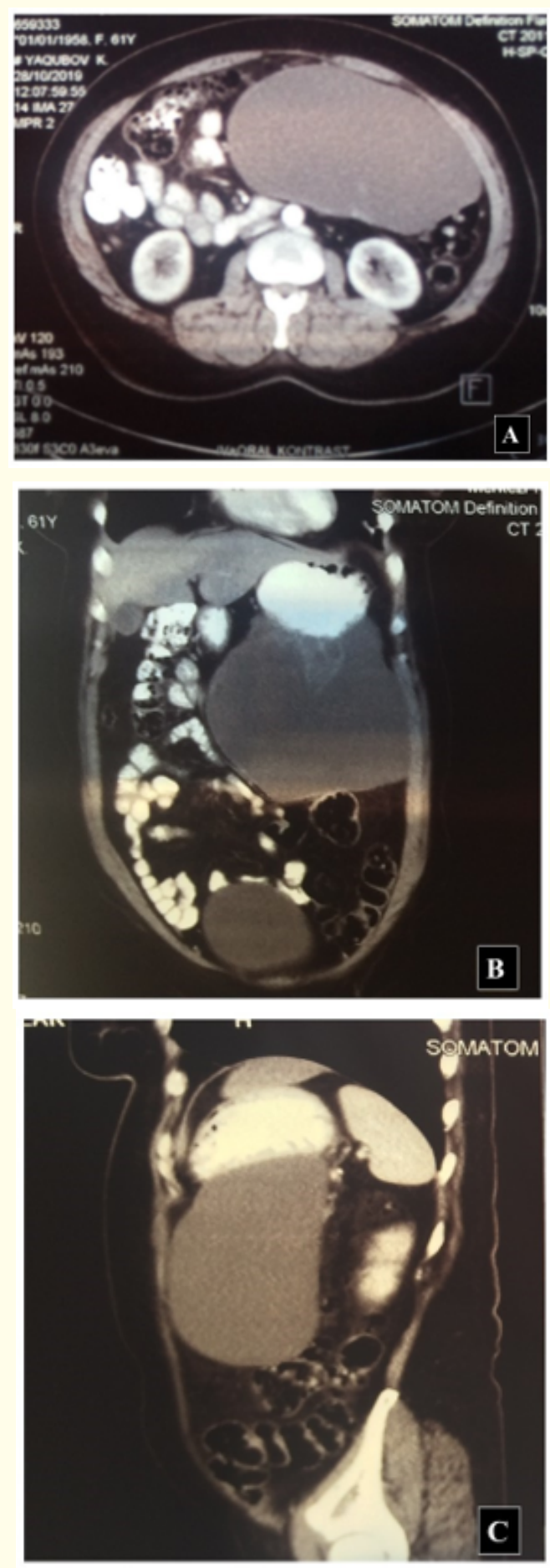

Figure : CT appearance (A- axial, B- coronal and C- sagittal planes) of cystic GIST in our patient. 


\section{Discussion and Conclusion}

Although GISTs are relatively uncommon $(<1 \%$ of all gastrointestinal neoplasms) compared to gastric adenocarcinoma, they are the most common mesenchymal tumors of the gastrointestinal tract [2,3]. GISTs were originally considered to be of smooth muscle (leiomyoma/leiomyosarcoma) or neural (schwannoma) origin, due to the appearance of these lesions on hematoxylin and eosin sections [12,17]. However, when electron microscopy became available, it became evident that these tumors had no evidence of muscle or schwannian differentiation ultrastructurally. This led to the non-specific term "stromal tumor". With the advent of immunohistochemical stains, these spindle cell lesions were shown to lack neural or myoid differentiation [17]. Therefore, only recently, with the help of immunohistochemistry, GISTs have been considered a distinct entity. These tumors are believed to arise from the Kit (CD117) positive interstitial cells of Cajal, the pacemaker cells of the GI tract [1,9,12-14]. Most GISTs express CD117 (90 - 95\%) and CD34 (70\%) $[9,15,19]$ and have KIT mutations, typically affecting the juxtamembrane domain encoded by exon 11 that normally inhibits the kinase activation loop in the absence of ligand binding $[7,24]$.

$50-60 \%$ of GISTs arise in the stomach. Small bowel lesions comprise $20-30 \%$ of the total, colonic less than $10 \%$, esophageal $1 \%$ and extra-gastrointestinal $5 \%$ [17]. It affects both sexes equally and $80 \%$ occur in persons over 50 years [7]. Microscopically, GISTs have a broad morphological spectrum. Three main histological subtypes have been widely accepted and they are spindle cell type (most common, 70\%), epithelioid type (20 - 25\%) and mixed spindle cell and epithelioid type [4]. About two-thirds of GISTs are malignant [1]. Independent adverse prognostic factors are large tumors, high mitotic count, non-gastric location, rupture and male gender $[20,21]$.

GISTs can be detected in variable sizes. The largest gastrointestinal stromal tumor ever recorded in literature weighed approximately $18.5 \mathrm{~kg}$ and measured $42.0 \times 31.0 \times 23.0 \mathrm{~cm}$ [1]. GISTs often present solid tumors and are rarely predominantly cystic $[7,18]$. GISTs with cystic changes are more frequently seen in high-grade malignancies. Because of aggressive tumor growth (in rapidly growing malignant GISTs), necrosis, liquefaction, and cystic degeneration occur from lack of adequate blood supply, leading to formation of large cystic spaces [10]. With other words complex cystic structure of GIST is characteristic for high-grade malignancies [18].

As mentioned above cystic GIST lesions are rare and invariably diagnosed late after excision. De Vogelaere., et al. (2012) reported a large $(12 \mathrm{~cm})$ exophytic unilocular cystic tumor with several membranous septa without accurate preoperative clinicoradiologic diagnosis of its origin. During surgery, the large mass was found to be attached by a narrow stalk to the large curvature of the stomach. After excision with wedge resection of the greater curvature of the stomach histopathological examination revealed GIST originated from the stomach. The authors suggested that in cases of unusual exophytic pendulated tumor of the stomach, always consider of GIST [15].

Hansen., et al. (2014) reported a 74-year-old female patient who underwent a Roux-en-Y cyst-jejunostomy for cystic mass preoperatively considered as a pancreatic pseudocyst. Few weeks postoperatively, she developed several melena episodes without any pathology found during repeat upper GI endoscopy. She was surgically revised. The main diagnostic concern was a pancreatic cystic neoplasm. A $12.0 \times 8.0 \times 5.0 \mathrm{~cm}$ size retro-gastric lesion was resected and pathology report indicated GIST, which was previously misdiagnosed as a pancreatic pseudocyst [16].

Zhu., et al. (2015) reported an exophytic retro-gastric large cystic lesion $(17 \times 15 \times 16 \mathrm{~cm})$ primarily considered to be a mass in the body of the pancreas, which was closely attached to the gastric wall. Even after a CT scan and MRI a preoperative diagnostic dilemma kept on unresolved. It was thought to be a pancreas-related mass-cystadenocarcinoma or cystadenoma undergone canceration. After block excision of the multicystic and partly solid mass with spleen, greater omentum and the attached wall of the stomach the histopathological examination revealed GIST [22].

A large $(18 \times 17 \times 10 \mathrm{~cm})$ mobile cystic GIST of the stomach in our case was precisely diagnosed preoperatively and surgically removed with wedge resection of the stomach. The tumor expressed CD 117 and mitotic index was s 5/50 HPFs. According to the generally accepted classifications for GIST assessment criteria, the tumor was classified to be in the high risk group, for which the suggested treatment is tyrosine kinase inhibitors. So, our patient was administrated imatinib for 12 months. The patient has no signs of recurrence and metastases during 16 months' postoperative period. 
One of the prominent features of our case was the appearance of a well-defined unilocular cystic mass with very small solid component as a pedicle that appeared to be extension of the stomach wall. Besides this is one of the rare cases of unilocular giant cystic GIST of the stomach diagnosed precisely in the preoperative period based on clinicoradiological examinations. In summary, GISTs with cystic appearance should be considered in the differential diagnosis of cystic abdominal tumors located in the supracolic compartment of the peritoneal cavity.

\section{Bibliography}

1. Mohamed A., et al. "Gigantic GIST: a case of the largest gastrointestinal stromal tumor found to date". Case Reports Surgery (2018): 6179861.

2. Nishida T and Hirota S. "Biological and clinical review of stromal tumours in the gastrointestinal tract". Histology and Histopathology 15.4 (2000): 1293-1301.

3. Tran T., et al. "The epidemiology of malignant gastrointestinal stromal tumors: an analysis of 1,458 cases from 1992 to 2000 ". American Journal of Gastroenterology 100.1 (2005): 162-168.

4. Zhao X and Yue Ch. "Gastrointestinal stromal tumor". Journal of Gastrointestinal Oncology 3.3 (2012): 189-208.

5. Ferreira SS., et al. "Synchronous gastrointestinal stromal tumors (GIST) and other primary cancers: Case series of a single institution experience". International Journal of Surgery 8.4 (2010): 314-317.

6. Demetri GD., et al. "NCCN task force report: update on the management of patients with gastrointestinal stromal tumors". Journal of the National Comprehensive Cancer Network 8.2 (2010): S1-S41.

7. Hamza AM., et al. "Gastrointestinal stromal tumour masquerading as a cyst in the lesser sac". BMJ Case Reports (2016): bcr2016-215479.

8. Okano H., et al. "A case of a stomach gastrointestinal stromal tumor with extremely predominant cystic formation". Clinical Journal of Gastroenterology 8.4 (2015): 197-201.

9. Wang L., et al. "Giant gastrointestinal stromal tumor with predominantly cystic changes: a case report and literature review". World Journal of Surgical Oncology 15 (2017): 220-223.
10. Levy AD., et al. "Gastrointestinal stromal tumors: radiologic features with pathologic correlation". Radiographics 23.2 (2003): 283-304.

11. DeMatteo R P., et al. "Two hundred gastrointestinal stromal tumors: recurrence patterns and prognostic factors for survival". Annals of Surgery 231.1 (2000): 51-58.

12. Hirota S., et al. "Gain-of-function mutations of c-kit in human gastrointestinal stromal tumors". Science 279.5350 (1998): 577-580.

13. Miettinen M and Lasota J. "Gastrointestinal stromal tumorsdefinition, clinical, histological, immunohistochemical, and molecular genetic features and differential diagnosis". Virchows Archiv 438.1 (2001): 1-12.

14. Koelz M., et al. "The impact of c-kit mutations on histomorphological risk assessment of gastrointestinal stromal tumors". European Surgery 39.1 (2007): 45-53.

15. De Vogelaere K., et al. "Unusual Appearance of a Pendulated Gastric Tumor: Always Think of GIST". Case Reports in Surgery (2012): 815941.

16. Hansen CA., et al. "Gastrointestinal stromal tumor (GIST) mistaken for pancreatic pseudocyst-case report and literature review". Clinical Case Reports 2.5 (2014): 197-200.

17. Streutker CJ., et al. "Interstitial cells of Cajal in health and disease. Part II: ICC and gastrointestinal stromal tumours". Histopathology 50.2 (2007): 190-202.

18. Miettinen M., et al. "Pathology and diagnostic criteria of gastrointestinal stromal tumors (GISTs): a review". European Journal of Cancer 38.5 (2002): S39-S51.

19. Cappellani A., et al. "Giant gastrointestinal stromal tumor (GIST) of the stomach cause of high bowel obstruction: surgical management". World Journal of Surgical Oncology 11 (2013): 172-176.

20. Joensuu H., et al. "Risk of recurrence of gastrointestinal stromal tumour after surgery: an analysis of pooled populationbased cohorts". Lancet Oncology 13.3 (2012): 265-274.

21. DeMatteo RP., et al. "Two hundred gastrointestinal stromal tumors: recurrence patterns and prognostic factors for survival”. Annals of Surgery 231.1 (2000): 51-58. 
22. Zhu CC., et al. "Exophytic gastrointestinal stromal tumor with cystic changes: a case report". Oncology Letters 7.5 (2014): 1427-1429.

23. Kumar A., et al. "Atypical presentation of gastrointestinal stromal tumor masquerading as a large duodenal cyst: a case report". International Journal of Surgery Case Reports 9 (2015): 123-126.

24. Joensuu H and DeMatteo RP. "The management of gastrointestinal stromal tumors: a model for targeted and multidisciplinary therapy of malignancy". Annual Review of Medicine 63 (2012): 247-258.

Volume 4 Issue 6 June 2021

(C) All rights are reserved by Ramiz B Bayramov., et al. 